\title{
Inner retinal layer change in glaucoma patients receiving anti-VEGF for neovascular age related macular degeneration
}

\author{
Rafidah Saleh $^{1}$ • Aashraya Karpe ${ }^{1}$ - Martin S. Zinkernagel ${ }^{1,2,3} \cdot$ Marion R. Munk ${ }^{1,3}$
}

Received: 1 November 2016 /Revised: 27 December 2016 / Accepted: 11 January 2017

(C) Springer-Verlag Berlin Heidelberg 2017

\begin{abstract}
Purpose The purpose was to evaluate the effects of long-term anti-VEGF treatment on the retinal nerve fiber layer (RNFL) and retinal ganglion cell layer (RGCL) thickness for patients with neovascular AMD and glaucoma.

Methods Medical records of respective patients who had received more than 15 anti-VEGF injections were reviewed. Initial and latest SD-OCT macular scans were segmented and changes of the RNFL and RGCL thickness at the four outer ETDRS quadrants were evaluated. Secondary outcome measures included changes of visual field parameters seen in automated perimetry.

Results Sixteen patients were included (mean age 78 \pm 6 years). The mean total number of anti-VEGF injections was $39 \pm 16$. The mean treatment duration was $6.1 \pm 2.1$ years. The mean IOP decreased from $18 \pm 5 \mathrm{mmHg}$ at baseline to 15 $\pm 5 \mathrm{mmHg}$ at the last visit $(p=0.026)$. The mean RNFL thickness volume of the outer ETDRS quadrants $\left(0.98 \pm 0.18 \mathrm{~mm}^{3}\right.$ to $\left.0.97 \pm 0.18 \mathrm{~mm}^{3} p=0.61\right)$ and its average thickness (37.9
\end{abstract}

Electronic supplementary material The online version of this article (doi:10.1007/s00417-017-3590-4) contains supplementary material, which is available to authorized users.

Marion R. Munk

marion_munk@hotmail.com

1 Department of Ophthalmology, Inselspital, Bern University Hospital, and University of Bern, Bern, Switzerland

2 Department of Clinical Research, University Hospital Bern, Bern, Switzerland

3 Bern Photographic Reading Center, Department of Ophthalmology, Inselspital, Bern University Hospital, and University of Bern, Bern, Switzerland $\pm 7.3 \mu \mathrm{m}$ to $37.2 \pm 7.4 \mu \mathrm{m}, p=0.6)$ did not significantly change. However, the average RGCL thickness decreased significantly from $0.86 \pm 0.12 \mathrm{~mm}^{3}$ to $0.79 \pm 0.11 \mathrm{~mm}^{3}$ $(p=0.01)$, and from $27.7 \pm 4.2$ to $25.9 \pm 3.7 \mu \mathrm{m}(p=0.01)$. Number of injections correlated with the RGCL change $(\mathrm{r} 2=0.36, p=0.01)$. The mean sensitivity, mean defect and absolute scotomata did not significantly change with $p$-values of $0.28,0.21$ and 0.07 , respectively.

Conclusion Patients under long term treatment with antiVEGF and concurrent glaucoma show significant decrease in macular RGLC volume. However, this decrease is comparable to reported RGCL decrease in patients under anti-VEGF treatment without underlying glaucoma and suggests that glaucoma patients may not be at a higher risk for losing macular RNFL and RGCL, at least if adequate control of intraocular pressure is maintained.

Keywords Retinal nerve fiber layer thickness - Ganglion cell layer thickness · Anti-VEGF · Wet AMD · Glaucoma · Exudative age-related macular degeneration $\cdot$ Intraocular pressure $\cdot$ Ocular hypertension $\cdot$ Visual fields $\cdot$ Retinal layer segmentation

\section{Introduction}

Neovascular age-related macular degeneration (nAMD) is an increasing burden in developed countries. This has made intravitreal injections of anti-vascular endothelial growth factor (anti-VEGF) agents an increasingly popular treatment method. The anti-VEGF agents approved for clinical use such as ranibizumab and aflibercept are known for their good safety profile and their efficacy treating VEGF-mediated diseases [1-3]. However continuous frequent injections, often over many years are needed to maintain satisfying visual function. 
The fact that any intravitreal injection transiently elevates the intraocular pressure has raised some concerns especially in patient populations with already pre-existing glaucomatous damages [4]. In fact, according to Kim et al. the IOP of eyes with a history of glaucoma takes longer to normalize after intravitreal drug administration [5]. Beside this well-known transient effect, a sustained elevation of the IOP has been described in about $10 \%$ of the treated patient population [6]. It is well known that the level of IOP is a strong risk factor for the development and/ or the progression of glaucoma; and that there is a positive correlation of the IOP with retinal ganglion cell (RGC) damage [7]. Even though glaucoma is diagnosed based on characteristic optic nerve cupping with corresponding visual field (VF) defects, significant loss of the RGC can occur before detectable VF defects are evident. Structural damage can precede detectable functional loss by up to 5 years [8-10]. Loss of the RGC and their axons is also known to occur in the posterior pole, where these cells may constitute 30 to $35 \%$ of the retinal thickness of the macular region [11]. Using OCT and retinal segmentation tools, it is now possible to quantify respective layers either manually or (semi)automatically $[12,13]$. Since then, several studies have been conducted to evaluate the potential damaging effect of long term intravitreal anti-VEGF injections on RGC by assessing the RNFL thickness in neovascular AMD patients. Interestingly most of these studies did not find a significant change in the RNFL thickness in neovascular AMD patients $[14,15]$. There was only one study done by Rimayanti et al. which compared ganglion cell complex (GCC) parameters and the thickness of the peripapillary retinal nerve fiber layer (RNFL) between normal eyes, eyes with AMD and eyes with AMD and glaucoma [16]. They found that the GCC was significantly different between the normal population and the AMD patients and that the RNFL was significantly different between the AMD population and AMD patients with concomitant glaucoma. Unfortunately, their study was only cross sectional and no follow-up; no long-term data on the course of the RNFL and GCC thickness in respective groups were available.

The aim of this study was to longitudinally evaluate the effects of long-term administration of intravitreal anti-VEGF on the macular RNFL and retinal ganglion cell layer (RGCL) thickness in patients with neovascular AMD and glaucoma, which in turn would address the question whether glaucoma patients under long-term anti-VEGF therapy require additional precautions such as IOP lowering therapy before intravitreal injections.

\section{Materials and methods}

This is a retrospective study of patients who had received more than 15 intravitreal injections of ranibizumab or/and aflibercept at the Department of Ophthalmology in the University Hospital of Bern, Switzerland. Due to its retrospective design, the study was granted a waiver to obtain informed consent.

All medical records of patients who had the diagnosis of neovascular AMD receiving anti-VEGF therapy between 2006 and 2015 were identified and only those with concurrent glaucoma were included. The diagnosis of glaucoma was either preexisting or the patient was diagnosed by a specialized consultant during the course of the intravitreal treatment. The inclusion criteria for glaucoma included either/or: IOP more than $21 \mathrm{mmHg}$, and/or a cup to disc ratio (CDR) 0.5 or more with either glaucomatous pattern visual field changes or OCT RNFL abnormality or both. Patients with diabetes, retinal vein occlusions, high myopia, posterior staphyloma, poor image quality, previous vitreoretinal surgeries and with absence of visual fields were excluded. If both eyes were eligible for the study, the eye more severely affected by glaucoma was chosen. If both eyes were equal, one of the eyes was chosen randomly.

Until 2013 patients received a ranibizumab loading dose followed by a capped pro-re nata (PRN) regimen with monthly visits and mandatory quarterly injections until July 2013 [17]. After that, all patients were treated according to the Bern treat and extend treatment regimen [18] using either aflibercept or ranibizumab. No paracentesis was performed in any of the included patients with the goal to alter the intra/postprocedural increase of IOP [19]. Visual function was tested by counting fingers in all patients immediately after the injection procedure.

The parameters analyzed included best corrected visual acuity (BCVA) ETDRS letter score at $4 \mathrm{~m}$, anterior segment and posterior segment findings, intraocular pressure (IOP) using Goldmann applanation tonometry at a fixed 3-month interval. OCT macular scans were performed using the Heidelberg spectral Domain (Heidelberg Engineering, Germany) each time the patient received an intravitreal antiVEGF injection. The images were acquired using image alignment eye-tracking software (TruTrack; Heidelberg Engineering Inc.) to obtain volumetric retinal scans with 49 parallel B-scans consisting of 512 A-scans separated by $121 \mu \mathrm{m}$ covering an area of $20^{\circ} \times 20^{\circ}$, whereby each B-scan was averaged nine times (automated real time repetition rate $=9$ ). By using the follow-up function, the exact protocol was repeated at every visit at the same retinal location.

Baseline was defined as the time-point when the intravitreal therapy was commenced. For analyses, the initial and latest macular OCT scans were selected for automated macular OCT segmentations using Spectralis OCT software. In cases where intravitreal injections were started before Heidelberg SD-OCT was available, the initial SD-OCT performed was taken for our analyses.

For retinal segmentation, the inbuilt Heidelberg Eye Explorer version 1.9.10.0 (Heidelberg Engineering, Germany) was used to measure the macular RNFL and the 
RGCL thickness. Heidelberg Eye Explorer segments 11 different retinal boundaries: the inner limiting membrane (ILM), the boundaries between the RNFL and the RGCL, between the RGCL and the inner plexiform layer (IPL), between the IPL and the inner nuclear layer (INL), between the INL and the outer plexiform layer (OPL), between the OPL and the outer nuclear layer (ONL), the external limiting membrane (ELM), two photoreceptor layers (PR1/2), the retinal pigment epithelium (RPE), and the basal membrane (BM) with the underlying choroid. Based on this segmentation algorithm the area between the ILM and the RNFL segmentation line (= mean RNFL thickness) and the area between the RNFL and RGCL segmentation line (= mean RGCL thickness) was automatically calculated by the inbuilt software (Fig. 1).

Initially the automated inbuilt Heidelberg segmentation software was employed. In a second step, the retinal segmentations were edited manually by one author (R.S). Later, as a final step, the segmentations were reviewed and corrected if necessary by an independent expert grader certified by the Bern Photographic Reading Centre (BPRC). The total ETDRS volumes of RGCL and RNFL and the average thickness of the four outer ETDRS quadrants (superior, nasal, inferior, temporal and its average) were recorded at baseline and also at the most recent visit.

Standard Automated Perimetry (SAP) was done using the Octopus Visual Field Analyzer (Haag Streit). The parameters such as Mean Sensitivity (MS), Mean Defect (MD) and number of absolute scotomata were recorded at the earliest and also at the latest visit. Only patients with reliable visual fields were included in the study. Anti-glaucomatous medication used and changes in medication were evaluated as well.

\section{Statistics}

Statistical analyses were performed using SPSS and GraphPad Prism (IBM, SPSS statistics, Version 21, SPSS Inc., Chicago, USA and GraphPad Prism, Version 6, GraphPad software Inc., La Jolla, CA, USA). Data were analyzed using student's paired $T$-test and multivariable regression analyses. The significance level was set at alpha $\leq 0.05$. Data are given as mean $\pm \mathrm{SD}$.

\section{Results}

A total of 16 eyes of 16 Caucasian patients, seven males and nine females with a mean age of $78 \pm 6$ years at the start of the anti-VEGF therapy were included in the study. The mean BCVA using the ETDRS Chart was $62 \pm 15$ letters at the timepoint of treatment initiation, $63 \pm 15$ letters at the timepoint of the initial RNFL and RGCL measurements and $63 \pm 14$ letters at the last visit. The mean change of BCVA was not statistically significant $(p=0.82)$. The mean duration of treatment was $6.1 \pm 2.1$ years. The mean total number of administered anti-VEGF injections was $39 \pm 16$ with a mean of $26 \pm 15$ ranibizumab and $13 \pm 10$ aflibercept injections.

The mean duration between the two OCT measurements was $3.7 \pm 1.6$ years with a mean of $25 \pm 14$ intravitreal antiVEGF injections administered during this time period.

In six patients, glaucoma was diagnosed before the onset of neovascular AMD, while in the remaining ten patients; glaucoma was diagnosed during the anti-VEGF treatment. Three (19\%) had Pseudoexfoliative glaucoma (PXG), ten (62\%) Primary Open Angle, and three (19\%) had Normal-tension Glaucoma. In the patients without preexisting glaucoma, the mean duration between the initiation of anti-VEGF treatment and the onset of glaucoma was $2.4 \pm 2$ years. The mean IOP at baseline was $18 \pm 5 \mathrm{mmHg}$ whereas at the last visit the IOP was $15 \pm 5 \mathrm{mmHg}$ and this difference was statistically significant $(p=0.026)$. The MS, MD and number of absolute scotomata did not show any significant change over time (Table 1).

Following anti-VEGF injections, in the group with preexisting glaucoma, three patients required additional antiglaucomatous eye drops and one patient received additionally Selective Laser Trabeculoplasty (SLT) followed by a glaucoma filtration surgery for better IOP control; Of the ten patients

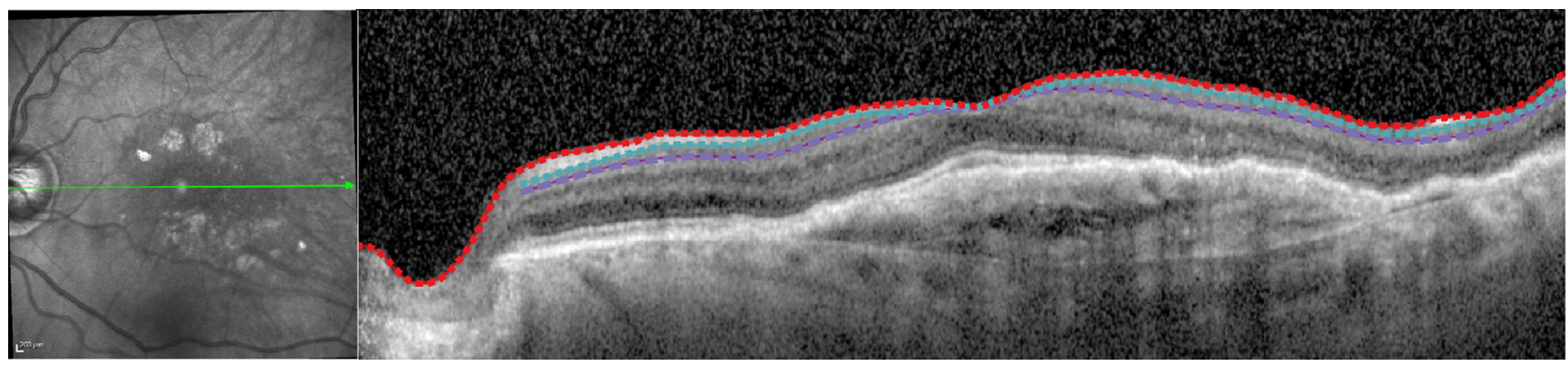

Fig. 1 Infrared image and retinal layer segmentation with optical coherence tomography (OCT); Infrared image of the macula of a 74year-old female with primary open angle glaucoma, where the OCT scan direction is shown as a green line. Representative OCT image of inner retinal layers, where the red, dotted line represents the internal limiting membrane, the turquoise dotted line represents the boundary between the retinal nerve fiber layer (RNFL) and the retinal ganglion cell layer (RGCL), and the purple, dotted line represents the boundary between RGCL and the inner plexiform layer 
Table 1 Mean changes of mean sensitivity, mean defect and absolute scotoma

\begin{tabular}{llll}
\hline Indices (mean $\pm \mathrm{SD})$ & Baseline & Latest follow-up & $p$-value \\
\hline Mean sensitivity (MS) & $17 \pm 9$ & $19 \pm 5$ & 0.28 \\
Mean defect (MD) & $6 \pm 4$ & $7 \pm 5$ & 0.21 \\
Absolute scotoma (n) & $4 \pm 6$ & $7 \pm 10$ & 0.07 \\
\hline
\end{tabular}

in the glaucoma treatment naïve group, nine patients needed to be started on anti-glaucomatous eye drops. For details regarding anti-glaucomatous therapy see supplemental Table 1.

The mean RNFL volume of the four outer ETDRS quadrants was $0.98 \pm 0.18 \mathrm{~mm}^{3}$ at baseline and $0.97 \pm 0.18 \mathrm{~mm}^{3}$ at the last visit, respectively $(p=0.61)$, while the mean average macular RNFL thickness of the same position at baseline was $37.9 \pm 7.3$ and $37.2 \pm 7.4 \mu \mathrm{m}$ at the last visit, respectively $(p=0.6)$ (Fig. 2, suppl. Fig. 1). A multivariable analysis for the potential effect of IOP at baseline, optic nerve head excavation at baseline and number of administered injections on RNFL change was not statistically significant $\left(R^{2}=0.24\right.$, $p=0.3$ ).

In contrast to the RNFL thickness, the RGCL of the four outer ETDRS quadrants decreased significantly during the observational period. The RGCL volume of $0.86 \pm 0.12 \mathrm{~mm}^{3}$ at baseline decreased to $0.79 \pm 0.11 \mathrm{~mm}^{3}(p=0.01)$, at the last visit, respectively, and the average thickness changed from $27.7 \pm 4.2 \mu \mathrm{m}$ at baseline to $25.9 \pm 3.7 \mu \mathrm{m}$, respectively $(p=$ 0.01 ), (Fig. 3). The number of administered injections between baseline and the last visit correlated with the RGCL change ( $\mathrm{r} 2=0.36, p=0.01$, Fig. 3, suppl. Fig. 2). The multivariable regression analysis showed a significant effect (in order of decreasing weight) for IOP at baseline, number of administered injections and optic nerve head excavation on RGCL change over time $\left(R^{2}=0.66, p=0.004\right.$, Table 2$)$.

\section{Discussion}

Since the introduction of intravitreal anti-VEGF injections, increase of IOP and concurrent loss of RNFL has been a concern for many years, especially taking into account that patients with neovascular AMD require long-term treatment to maintain their visual function. This concern is even more evident in patients with already pre-existing damage, and who may be more susceptible to IOP increase such as glaucoma patients. In fact, Kim et al. reported that the transient IOP increase is prolonged in glaucomatous eyes compared to eyes without glaucomatous co-morbidity [5]. It was also stated by Griffith and Goldberg that glaucoma patients with comorbid retinal disease had a higher prevalence of blindness and low vision than those without comorbid retinal disease [20]. However, Shin et al. concluded that multiple injection of
anti-VEGF did not lead to significant change in RNFL thickness in wet AMD, Diabetic Retinopathy (DR) and retinal vein occlusions (RVO) patients. IOP fluctuations and the frequency of the injections did not appear to adversely affect RNFL thickness. Decreased RNFL thickness, in fact, was associated with severity of retinal ischemia in the DR and RVO patients suggesting that inner retinal ischemia itself could be a cause of RNFL loss rather than anti-VEGF [21].

From our study of a patient population with neovascular AMD and concomitant glaucoma, we have found that there was a significant decrease of RGCL over the course of longterm anti-VEGF treatment, while the changes on macular RNFL were not significant. So far there are no other studies evaluating the changes of RGCL thickness in patients with neovascular AMD and glaucoma over time. However, there were other studies, which were done by Zucchiatti et al. and Lee and $\mathrm{Yu}$, that confirmed that the RGCL in ARMD is significantly lower than in the normal population $[22,23]$. Rimayanti et al., compared the Ganglion Cell Complex (GCC, which consists of the nerve fiber layer, ganglion cell layer and inner plexiform layer) and peripapillary RNFL thickness of three different patient groups which were healthy controls, AMD and AMD patients with glaucoma in a crosssectional study [16]. Interestingly, they found the average GCC thickness to be significantly decreased in AMD eyes compared to the healthy control eyes, whereas there was no difference between the AMD eyes with and without glaucoma [16]. A recent published manuscript from our group following the RGCL and RNFL thickness of neovascular AMD patients under long-term anti-VEGF reveal a comparable RGCL thickness decrease under long-term anti-VEGF compared to our patient population, which indicates that well-controlled glaucomatous patients may not be at a higher risk when receiving long-term anti-VEGF treatment and may not require additional precautions such as IOP-lowering therapy before intravitreal injections [24]. Another possibility is that rather AMD than anti-VEGF treatment may lead to RGCL loss, given that a recent study found out that untreated as well as dry AMD eyes show a reduction in RGCL thickness [22]. The fact that the number of injections correlated with the RGCL change suggests an association between anti-VEGF injections and RGCL loss, but it may just describe an indirect correlation while the direct association may be found between the duration of AMD and RGCL loss.

Age-related loss of RGCL thickness is documented to be around $0.3-0.6 \%$ per year, while the loss of RGCL thickness in our study was $8.5 \%$ over a mean of 3.7 years [25]. However, the visual field's parameters had no significant changes over time. This is in line with Quigley et al. who did postmortem analysis of enucleated human glaucomatous eyes and found that a $20 \%$ loss of RGC throughout the central $30^{\circ}$ of the retina is necessary to induce a $5-\mathrm{dB}$ sensitivity loss in automated perimetry, while a $40 \%$ loss results in a $10-\mathrm{dB}$ 

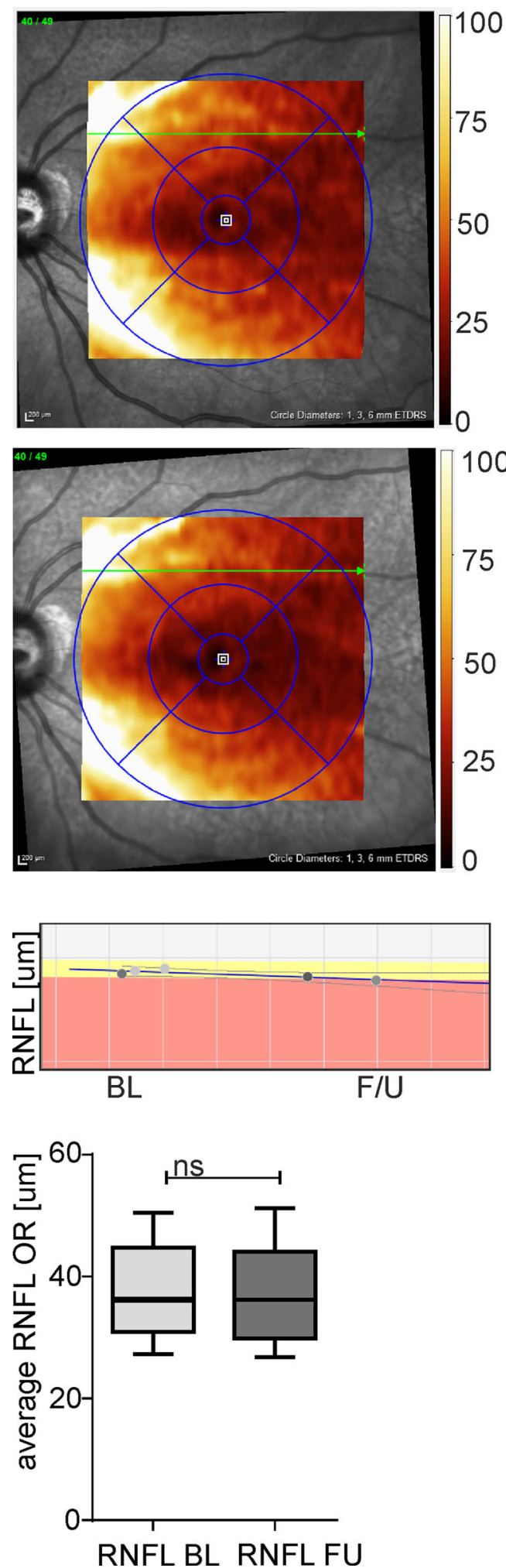

loss in sensitivity [9]. While the increase in absolute scotomata in our patient group may be explained by the natural course of neovascular AMD with loss of macular photoreceptors, the increase of the Mean Defect, which reflects the overall depression of the field, may be attributed to RGCL loss [26]. The
Fig. 2 Effect of continuous anti-vascular endothelial growth factor (VEGF) treatment on the retinal nerve fiber layer (RNFL) in patients with glaucoma. Co-localized infrared image of a representative patient with average thickness of the retinal nerve fiber layer (RNFL) in each quadrant at baseline (top) and follow-up (bottom) and RNFL thickness development of the outer ring (OR) from baseline to follow-up (middle panel). Box-and-whisker plots of RNFL thickness at baseline and followup (bottom) ( $n=16, n s$ not significant)

loss of RGCL thickness in our data may be due to the underlying glaucoma, the administration of anti-VEGF agents or due to the disease itself given that the multivariable analyses identified IOP at baseline and the number of administered injections for having the strongest effect on RGCL change over time. Glaucoma preferentially affects the innermost retinal layers: the nerve fiber, ganglion cell and inner plexiform layers, which contain, respectively, the axons, cell bodies and dendrites of the ganglion cells, and this is also true for AMD [27-29]. Both AMD and glaucoma share a common pathogenesis of neuronal cell loss via apoptosis [7, 30]. Therefore, several studies have proven the importance of the evaluation of the RGCL thickness in glaucoma eyes as this parameter not only displays progression of respective disease but was also shown to stronger correlate with visual field sensitivity than the peripapillary RNFL thickness [31]. Tan et al. who studied the GCC thickness in glaucoma confirmed that macular imaging with Fourier-domain (FD)-OCT is a useful method for glaucoma diagnosis and has the potential for tracking glaucoma progression. They also claimed that the improved diagnostic power of macular GCC imaging is on par with, and complimentary to, peripapillary RNFL imaging [32]. Kim et al. also agreed that macular GCC thickness and RNFL thickness showed similar diagnostic performance for detecting early, moderate and severe glaucoma [33]. As for AMD, studies in the previous literature too have found a significant RGCL complex thickness decrease in neovascular AMD eyes as well as in patients suffering from dry AMD [22].

The mean physiological loss of RNFL thickness is around $0.18 \mu \mathrm{m} /$ year [34, 35]. However, our analyses did not find any significant loss in the macular RNFL during the observational period. Since we concentrated on the RNFL thickness in the macular region in contrast to most of the other studies which evaluated the peripapillary RNFL, we are unable to directly compare the change of macular RNFL with previous published reports. The best comparable data are provided by a recent published study of our group. This study did not find a significant decrease of the macular RNFL in neovascular AMD eyes treated with around 32 anti-VEGF injections over a period of 45.3 months [24]. Further previously published studies did not notice any significant alteration in the peripapillary RNFL thickness post anti-VEGF. According to Demirel et al., long-term treatment with anti-VEGF agents did not lead to significant changes in peripapillary RNFL thickness in wet AMD. Their follow-up was about 3 years 


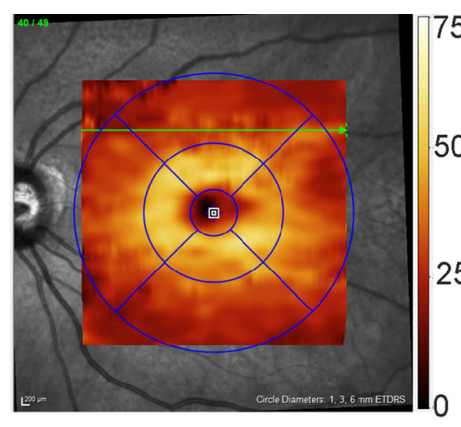

75
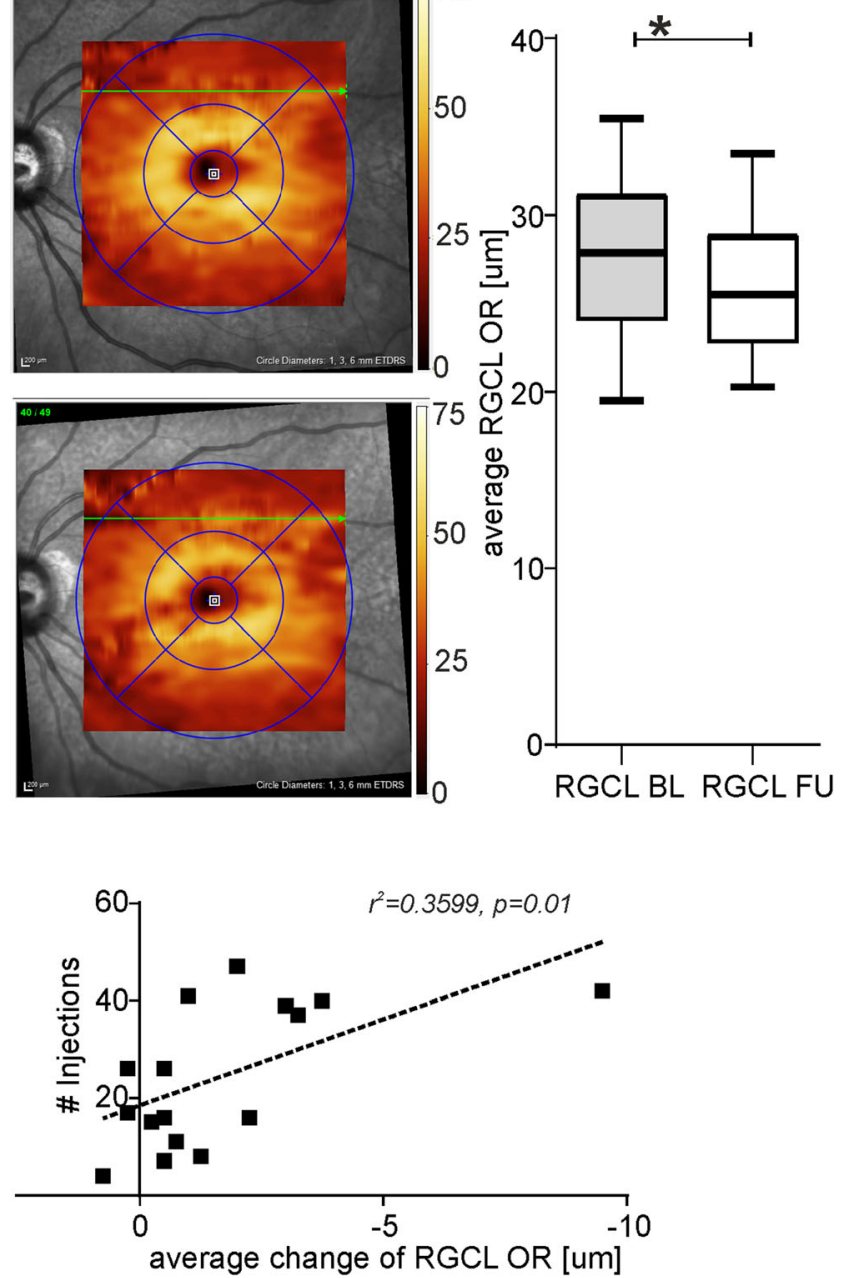

Fig. 3 Effect of continuous anti-vascular endothelial growth factor (VEGF) treatment on the retinal ganglion cell layer (RGCL) in patients with glaucoma. Co-localized infrared image of a representative patient with average thickness of the retinal ganglion cell layer (RGCL) in each quadrant at baseline (top) and follow up (bottom). Box-and-whisker plots of RGCL thickness of the outer ring (OR) at baseline and follow up (right) $(n=16, *=p<0.05)$. Scatter plot with the correlation between RGCL thickness change in the OR and number of injections (Pearson correlation)

with a mean of 13 injections [14]. Concordantly, El-Ashry et al. stated that repeated ranibizumab injections in wet AMD appear to have no harmful effect on the peripapillary RNFL thickness, at least in the short-term, given that this paper focused on the effects of the loading dose of three intravitreal anti-VEGF injections, and also Horsely et al. concluded that long-term anti-VEGF agents did not lead to significant changes in RNFL thickness in neovascular AMD population. Their longest follow-up was about 2 years (27 months), and the highest number of injections was 17.7 [15]. Another study done by Yuda et al. also noted no relationship between neovascular AMD and peripapillary RNFL thickness [29]. Thus, the abovementioned studies are in line with our recent findings.
Table 2 The predictive parameters for retinal ganglion cell layer (RGCL) average thickness change analyzed with a multivariable regression analysis

Retinal ganglion cell layer (RGCL) average thickness change

\begin{tabular}{llll}
\hline Evaluated factor & Partial $\mathrm{Eta}^{2}$ & Model $R^{2}$ & $p$-value \\
\hline IOP & 0.47 & 0.66 & 0.004 \\
N of injections & 0.15 & & \\
ONH excavation & 0.065 & & \\
\hline
\end{tabular}

$I O P$ intraocular pressure at baseline, $O N H$ optic nerve head

So far there was only one study done by Martinez-de-laCasa et al., who noted a significant peripapillary RNFL loss in ARMD patients receiving intravitreal anti-VEGF after a 12 months follow-up [28]. In the study performed by Rimayanti et al., the peripapillary RNFL was not significantly different between AMD and normal healthy control eyes, however glaucomatous AMD eyes showed a significantly thinner RNFL than the AMD eyes not suffering from glaucoma [16].

How RGCL changes can occur without concomitant RNFL loss remains speculative at this point as one would assume that the loss of a ganglion cell would lead to associated loss of its axon. One explanation may be that, since the thickness and volume changes are studied in the outer macular subfields where the RNFL contains fibers from the ganglion cells directly subjacent to the RNFL as well as from ganglion cells in areas farther away, the RNFL in these respective subfields are more redundant and supernumerary compared to the RGCL. This implicates that changes in the RNFL may not only be much smaller but also lag behind those of the RGCL and need a much longer time to be detected.

Thus, according to the literature available it remains unknown, whether the blockage of VEGF, which is known for its neurotrophic properties or the transient IOP increase directly after the injection procedure definitely affect the RNFL thickness. In order to solve this question, a long-term prospective study including an age-matched control group receiving an equal volume of intravitreal saline would be required. However, due to ethical reasons this is difficult to obtain. Our patient population consisting of patients suffering from glaucoma as well as from neovascular AMD receiving longterm anti-VEGF demonstrate that with effective control of the IOP, no significant RNFL loss was found over a mean period of 3.7 years. However, our patient population may not be representative for patients suffering from progressive or severe glaucoma as most of the patients were well managed, and the IOP were very well controlled.

The limitations of this study include its retrospective design and the small patient population, and, as such, it may be underpowered to detect differences in visual field parameters. The difficulty in segmentation of the retinal layers due to the 
irregular contour of retina in AMD as well as the poor demarcation of certain layers may contribute to errors which can serve as confounding factors in the final result [36, 37]. Although a multivariable regression analysis was included to determine effect variables, and a previous paper of our group including patients with neovascular AMD under long-term anti-VEGF treatment without glaucoma matched in terms of age and number of administered injection served as a comparable "control" group, this study lacks a control group of AMD patients without glaucoma on long-term anti-VEGF and a group of AMD patients without treatment in order to explore how much of these changes reflect the natural progression and how much can be attributed to glaucoma progression, AMD and anti-VEGF injections.

To summarize, this is the first study to longitudinally follow macular RGCL and RNFL thickness in glaucomatous patients with neovascular AMD under long-term treatment. This study provides first evidence that well-managed glaucomatous patients under long-term anti-VEGF treatment may not be at a higher risk for losing macular RNFL and RGCL than "usual" neovascular AMD patients. However, more conclusive evidence needs to be obtained with a larger sample size and a prospective study design.

\section{Compliance with ethical standards}

Funding No funding was received for this research. Martin S Zinkernagel is a stock holder and a consultant for Novartis and a consultant for Bayer. Marion R Munk is a consultant for Novartis and Bayer and received travel grants from Bayer. The remaining authors certify that they have no affiliations with or involvement in any organization or entity with any financial interest (such as honoraria; educational grants; participation in speakers' bureaus; membership, employment, consultancies, stock ownership, or other equity interest; and expert testimony or patent-licensing arrangements), or non-financial interest (such as personal or professional relationships, affiliations, knowledge or beliefs) in the subject matter or materials discussed in this manuscript.

Ethical approval All procedures performed in studies involving human participants were in accordance with the ethical standards of the institutional and/or national research committee and with the 1964 Helsinki Declaration and its later amendments or comparable ethical standards. For this type of study formal consent is not required.

\section{Financial support None.}

Conflict of interest The authors have no conflict of interest to report.

\section{References}

1. Bressler SB, Almukhtar T, Bhorade A, Bressler NM, Glassman AR, Huang SS, Jampol LM, Kim JE, Melia M, Diabetic Retinopathy Clinical Research Network Investigators (2015) Repeated intravitreous ranibizumab injections for diabetic macular edema and the risk of sustained elevation of intraocular pressure or the need for ocular hypotensive treatment. JAMA Ophthalmol 133:589-597. doi:10.1001/jamaophthalmol.2015.186
2. Schmidt-Erfurth U, Eldem B, Guymer R, Korobelnik JF, Schlingemann RO, Axer-Siegel R, Wiedemann P, Simader C, Gekkieva M, Weichselberger A, EXCITE Study Group (2011) Efficacy and safety of monthly versus quarterly ranibizumab treatment in neovascular age-related macular degeneration: the EXCITE study. Ophthalmology 118:831-839. doi:10.1016/j.ophtha.2010.09.004

3. Holz FG, Amoaku W, Donate J, Guymer RH, Kellner U, Schlingemann RO, Weichselberger A, Staurenghi G, SUSTAIN Study Group (2011) Safety and efficacy of a flexible dosing regimen of ranibizumab in neovascular age-related macular degeneration: the SUSTAIN study. Ophthalmology 118:663-671. doi:10.1016/j.ophtha.2010.12.019

4. Good TJ, Kimura AE, Mandava N, Kahook MY (2011) Sustained elevation of intraocular pressure after intravitreal injections of anti-VEGF agents. Br J Ophthalmol 95:1111-1114. doi:10.1136/bjo.2010.180729

5. Kim JE, Mantravadi AV, Hur EY, Covert DJ (2008) Short-term intraocular pressure changes immediately after intravitreal injections of anti-vascular endothelial growth factor agents. Am J Ophthalmol 146:930-934 e931. doi:10.1016/j.ajo.2008.07.007

6. Dedania VS, Bakri SJ (2015) Sustained elevation of intraocular pressure after intravitreal anti-VEGF agents: what is the evidence? Retina 35:841-858. doi:10.1097/IAE.0000000000000520

7. Quigley HA (1999) Neuronal death in glaucoma. Prog Retin Eye Res 18:39-57

8. Sommer A, Katz J, Quigley HA, Miller NR, Robin AL, Richter RC, Witt KA (1991) Clinically detectable nerve fiber atrophy precedes the onset of glaucomatous field loss. Arch Ophthalmol 109:77-83

9. Quigley HA, Dunkelberger GR, Green WR (1989) Retinal ganglion cell atrophy correlated with automated perimetry in human eyes with glaucoma. Am J Ophthalmol 107:453-464. doi:10.1016/0002-9394(89)90488-1

10. Airaksinen PJ, Drance SM, Douglas GR, Mawson DK, Nieminen H (1984) Diffuse and localized nerve fiber loss in glaucoma. Am J Ophthalmol 98:566-571

11. Zeimer R, Asrani S, Zou S, Quigley H, Jampel H (1998) Quantitative detection of glaucomatous damage at the posterior pole by retinal thickness mapping: a pilot study. Ophthalmology 10:224-231

12. Chiu SJ, Li XT, Nicholas P, Toth CA, Izatt JA, Farsiu S (2010) Automatic segmentation of seven retinal layers in SDOCT images congruent with expert manual segmentation. Opt Express 18: 19413-19428. doi:10.1364/OE.18.019413

13. Ishikawa H, Stein DM, Wollstein G, Beaton S, Fujimoto JG, Schuman JS (2005) Macular segmentation with optical coherence tomography. Invest Ophthalmol Vis Sci 46:2012-2017. doi:10.1167/iovs.04-0335

14. Demirel S, Batioglu F, Ozmert E, Erenler F (2015) The effect of multiple injections of ranibizumab on retinal nerve fiber layer thickness in patients with age-related macular degeneration. Curr Eye Res 40:87-92. doi:10.3109/02713683.2014.917190

15. Horsley MB, Mandava N, Maycotte MA, Kahook MY (2010) Retinal nerve fiber layer thickness in patients receiving chronic anti-vascular endothelial growth factor therapy. Am J Ophthalmol 150:558-561 e551. doi:10.1016/j.ajo.2010.04.029

16. Rimayanti U, Kiuchi Y, Yamane K, Latief MA, Mochizuki H, Hirata J, Akita T, Tanaka J (2014) Inner retinal layer comparisons of eyes with exudative age-related macular degeneration and eyes with age-related macular degeneration and glaucoma. Graefes Arch Clin Exp Ophthalmol 252:563-570. doi:10.1007/s00417-0132496-z

17. MenkeMN,ZinkernagelMS, Ebneter A, WolfS (2014)Functional and anatomical outcome of eyes with neovascularage-related macular degeneration treated with intravitreal ranibizumab following an exit strategy regimen. Br J Ophthalmol 98:1197-1200. doi:10.1136/bjophthalmol-2013-304775 
18. Freund KB, Korobelnik JF, Devenyi R, Framme C, Galic J, Herbert E, Hoerauf H, Lanzetta P, Michels S, Mitchell P, Mones J, Regillo C, Tadayoni R, Talks J, Wolf S (2015) Treat-and-extend regimens with anti-VEGF agents in retinal diseases: a literature review and consensus recommendations. Retina 35:1489-1506. doi:10.1097/IAE.0000000000000627

19. Katayama BY, Bonini-Filho MA, Messias AM, Paula JS, Martin LF, Costa R, Jorge R (2014) Comparison of acetazolamide, brimonidine, and anterior chamber paracentesis for ocular hypertension control after initial intravitreal bevacizumab injection: a randomized clinical trial. J Glaucoma 23:461-463. doi:10.1097/IJG.0b013e3182948476

20. Griffith JF, Goldberg JL (2015) Prevalence of comorbid retinal disease in patients with glaucoma at an academic medical center. Clin Ophthalmol 9:1275-1284. doi:10.2147/OPTH.S85851

21. Shin HJ, Shin KC, Chung H, Kim HC (2014) Change of retinal nerve fiber layer thickness in variousretinal diseases treated with multiple intravitreal antivascular endothelial growth factor. Invest Ophthalmol Vis Sci 55:2403-2411. doi:10.1167/iovs.13-13769

22. Zucchiatti I, Parodi MB, Pierro L, Cicinelli MV, Gagliardi M, Castellino N, Bandello F (2015) Macular ganglion cell complex and retinal nerve fiber layer comparison in different stages of agerelated macular degeneration. Am J Ophthalmol 160:602-607 e601. doi:10.1016/j.ajo.2015.05.030

23. Lee EK, Yu HG (2015) Ganglion cell-inner plexiform layer and peripapillary retinal nerve fiber layer thicknesses in age-related macular degeneration. Invest Ophthalmol Vis Sci 56:3976-3983. doi:10.1167/iovs.15-17013

24. Beck M, Munk MR, Ebneter A, Wolf S, Zinkernagel MS (2016) Retinal ganglion cell layer change in patients treated with antivascular endothelial growth factor for neovascular age-related macular degeneration. Am J Ophthalmol 167:10-17. doi:10.1016/j. ajo.2016.04.003

25. Harwerth RS, Wheat JL, Rangaswamy NV (2008) Age-related losses of retinal ganglion cells and axons. Invest Ophthalmol Vis Sci 49:4437-4443. doi:10.1167/iovs.08-1753

26. Curcio CA, Medeiros NE, Millican CL (1996) Photoreceptor loss in age-related macular degeneration. Invest Ophthalmol Vis Sci 37: 1236-1249

27. Garas A, Papp A, Hollo G (2013) Influence of age-related macular degeneration on macular thickness measurement made with fourierdomain optical coherence tomography. J Glaucoma 22:195-200. doi:10.1097/IJG.0b013e31824083e6

28. Martinez-de-la-Casa JM, Ruiz-Calvo A, Saenz-Frances F, RecheFrutos J, Calvo-Gonzalez C, Donate-Lopez J, Garcia-Feijoo J
(2012) Retinal nerve fiber layer thickness changes in patients with age-related macular degeneration treated with intravitreal ranibizumab. Invest Ophthalmol Vis Sci 53:6214-6218. doi:10.1167/iovs.12-9875

29. Yuda K, Inoue Y, Tomidokoro A, Tamaki Y, Yanagi Y (2010) Nerve fiber layer thickness in exudative age-related macular degeneration in Japanese patients. Graefes Arch Clin Exp Ophthalmol 248:353359. doi:10.1007/s00417-009-1222-3

30. Dunaief JL, Dentchev T, Ying GS, Milam AH (2002) The role of apoptosis in age-related macular degeneration. Arch Ophthalmol 120:1435-1442

31. Shin HY, Park HY, Jung KI, Park CK (2013) Comparative study of macular ganglion cell-inner plexiform layer and peripapillary retinal nerve fiber layer measurement: structure-function analysis. Invest Ophthalmol Vis Sci 54:7344-7353. doi:10.1167/iovs.1312667

32. Tan O, Chopra V, Lu AT, Schuman JS, Ishikawa H, Wollstein G, Varma R, Huang D (2009) Detection of macular ganglion cell loss in glaucoma by Fourier-domain optical coherence tomography. Ophthalmology 116:2305-2314 e2301-2302. doi:10.1016/j. ophtha.2009.05.025

33. Kim NR, Lee ES, Seong GJ, Kim JH, An HG, Kim CY (2010) Structure-function relationship and diagnostic value of macular ganglion cell complex measurement using Fourier-domain OCT in glaucoma. Invest Ophthalmol Vis Sci 51:4646-4651. doi:10.1167/10.1167/iovs.09-5053

34. Girkin CA, McGwin G Jr, Sinai MJ, Sekhar GC, Fingeret M, Wollstein G, Varma R, Greenfield D, Liebmann J, Araie M, Tomita G, Maeda N, Garway-Heath DF (2011) Variation in optic nerve and macular structure with age and race with spectral-domain optical coherence tomography. Ophthalmology 118:2403-2408. doi:10.1016/j.ophtha.2011.06.013

35. Budenz DL, Anderson DR, Varma R, Schuman J, Cantor L, Savell J, Greenfield DS, Patella VM, Quigley HA, Tielsch J (2007) Determinants of normal retinal nerve fiber layer thickness measured by Stratus OCT. Ophthalmology 114:1046-1052. doi:10.1016/j. ophtha.2006.08.046

36. Patel PJ, Chen FK, da Cruz L, Tufail A (2009) Segmentation error in Stratus optical coherence tomography for neovascular agerelated macular degeneration. Invest Ophthalmol Vis Sci 50:399404. doi:10.1167/iovs.08-1697

37. Lee HJ, Kim MS, Jo YJ, Kim JY (2015) Ganglion cell-inner plexiform layer thickness in retinal diseases: repeatability study of spectral-domain optical coherence tomography. Am J Ophthalmol 160:283-289 e281. doi:10.1016/j.ajo.2015.05.015 